\title{
VARIATION IN HUMAN SWEET TASTE RECEPTOR MAY RESULT IN DIFFERENT LEVELS OF SWEET INTENSITY VARIABILITY BETWEEN SWEET STIMULI
}

by

\section{JAMES C. WAKSMONSKI}

B.S., University of Wisconsin-Madison, 2012

\begin{abstract}
A THESIS
submitted in partial fulfillment of the requirements for the degree
\end{abstract}

MASTER OF SCIENCE

Food Science

College of Agriculture

KANSAS STATE UNIVERSITY

Manhattan, Kansas

2016

Approved by:

Major Professor

Dr. Kadri Koppel 


\begin{abstract}
Understanding the physiological activation and genetic variation of the sweet taste receptor (T1R) can improve formula optimization for products intended for a population of genetically diverse people. Computer modeling and cell culture techniques have thoroughly described the structure and binding sites of the T1R. The structure contains two subunits (T1R2 and T1R3) with multiple domains where sweet molecules can interact. The interaction takes place between individual molecules and amino acid residues of the T1R. The residues with which individual molecules interact differs between sweeteners. Person-to-person differences in the residue sequence of the T1R can arise from variation in the genes that encode the T1R (TAS1R), potentially effecting the function of the receptor. As a result of the specificity of binding interactions, genetic variation may affect sensitivity to some sweeteners, while sensitivity to other sweeteners remains normal. Therefore, it can be hypothesized that the level of person-toperson sweetness sensitivity variation may differ for each sweetener depending on the binding site of the molecule and site of T1R variation. The T1R structure, binding sites, and genetic variation will be reviewed, as well as potential parameters to predict the degree of sensitivity variation and formulation strategies to minimize the effects of sensitivity variation.
\end{abstract}




\section{Table of Contents}

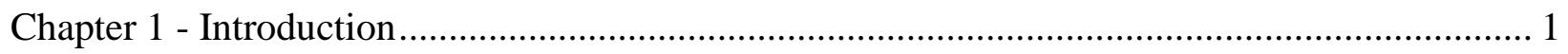

Chapter 2 - Sweet Taste Receptor (T1R) Structure and Binding Mechanisms ........................... 3

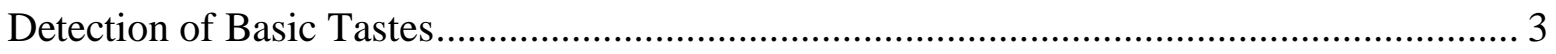

Structure and Activation of the T1R ......................................................................... 3

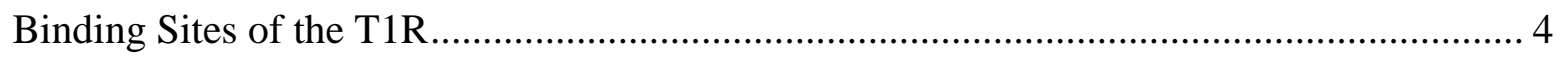

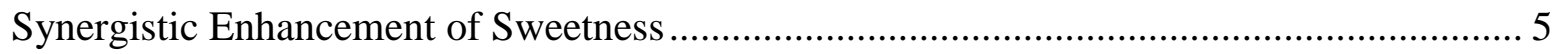

Chapter 3 - The Effect of Genetic Variation on Sensitivity to Sweet Tastants ......................... 10

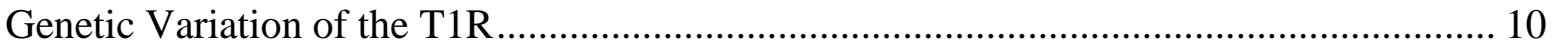

Effect of Genetic Variation on Sensitivity to Tastants Received by G-Coupled Protein

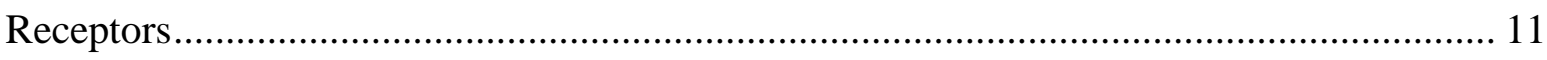

Genetic Variation and its Effect on Person-to-Person Sweetness Intensity Variability of

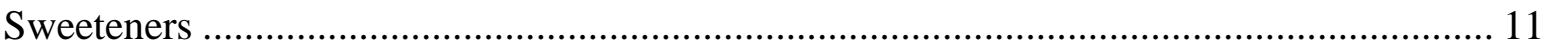

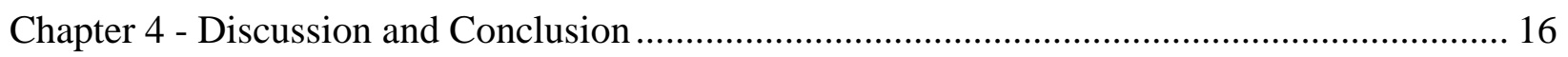

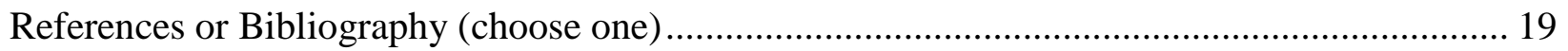

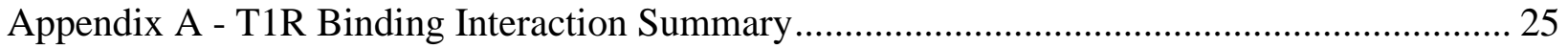

Summary of Binding Domains and Important Amino Acid Residues in the T1R ................ 25 


\section{Chapter 1 - Introduction}

With the increasing rate of obesity across the world (WHO, 2003; James, 2009), many consumers are looking for reduced caloric substitutions of the foods they currently consume. Calories can be reduced in a food with the removal of any of the calorie-containing macronutrients: fat, carbohydrates, and protein. In many sweet tasting food products, such as soda and chewing gum, reducing simple carbohydrates, or sugars, is the only realistic way to reduce calories. However, the taste and texture properties that sugars provide is challenging to replicate in sugar-reduced products. Despite a reduction in sugar content, consumers still have an expectation that the reduced- or sugar-free food be similarly sweet as the original product. Therefore, product developers often employ artificial and natural high potency sweeteners to provide the expected level of sweetness in a reduced- or sugar-free food without adding calories. However, high-potency sweeteners often carry off-flavors, affecting the taste and flavor of the sugar-reduced product (Allen et al., 2013; Kuhn et al., 2004; Li et al, 2015). The replacement of sugar in reduced- or sugar-free food is also challenging for the chemical interactions, physical bulking, and texture properties sugars participate in and provide. Some texture properties may be partially replicated by bulking ingredients, such as polydextrose, while supplying lower energy content by not being fully digested (Varzakas et al., 2012).

With an ever growing global food market, products are developed for increasingly genetically diverse populations. Genetic differences may affect sensitivity to certain sweeteners more than others and result in higher levels of sensitivity variation for specific sweeteners. Highpotency sweeteners have very different structures from the traditional mono- and disaccharides that humans have evolved to detect as a source of energy (Chandrashekar et al., 2006). Although both sugars and high-potency sweeteners are perceived as sweet, a departure from the 
evolutionary sweet tastant structures may lead to challenges with product formulation. Therefore, formula optimization may become problematic for foods that use certain sweeteners and are intended for a genetically diverse population of people.

This paper aims to propose that the amount of sweetness intensity delivered by some high potency sweeteners may vary more between genetically diverse people than the traditional mono- and disaccharides that humans have evolved to detect. In the processes of doing so, the structures involved in sweet taste detection, mechanisms and binding sites for sweet tastants, and genetic variation of sweet taste receptor will be reviewed. 


\section{Chapter 2 - Sweet Taste Receptor (T1R) Structure and Binding}

\section{Mechanisms}

\section{Detection of Basic Tastes}

Humans can perceive five basic tastes: sweet, salty, sour, bitter, and umami. The perception of these sensations is initiated by the activation of basic taste receptor cells in the oral cavity with corresponding molecules. Salty and sour tastants are received by ion channel receptor cells which allow for direct entry of $\mathrm{Na}^{+}$and $\mathrm{H}^{+}$ions, respectively (Lopez-Jimenez et al., 2006; Ishimaru et al., 2006; Huang et al., 2006; Chandrashekar et al., 2010). Sweet, bitter, and umami tastants interact with heterodimeric G-coupled protein receptor cells, which are categorized as Type 1 (T1R) and Type 2 (T2R) taste receptor cells (Hoon et al., 1999; Adler et al., 2000; Matsunami et al., 2000; Chandrashekar et al., 2000; Nelson et al., 2001, 2002; Li et al., 2002; Zhang et al., 2003; Zhao et al., 2003; Nie et al., 2005). The T1R is capable of detecting both sweet and umami tastants because it is made up of three subunit proteins: T1R1, T1R2, and T1R3. The T1R1 and T1R3 subunits detect umami tastants, while the T1R2 and T1R3 subunits detect sweet tastants (Nelson et al., 2001, 2002; Li et al., 2002; Zhang et al., 2003; Nie et al., 2005). The T2R detects bitter tastants with dozens of different subunits (Adler et al., 2000; Matsunami et al., 2000; Chandrashekar et al., 2000).

Each of the receptor cells are grouped together into taste buds in papillae throughout the oral cavity (Dasgupta et al., 1990; Kinnamon et al., 1993; Hoon et al., 1999). These papillae can be divided into four different categories: fungiform, filiform, circumvallate, and foliate. Although the filiform papillae do not contain receptor cells that participate in basic taste recognition, the other three classes of papillae each have varying proportions of each type of taste receptor cell (Hoon et al., 1999; Kim et al., 2003; Yarmolinsky et al., 2009). For example, 
T1R2 cells are more commonly expressed in fungiform papillae, located at the front of the tongue, and less commonly expressed in circumvallate and foliate papillae at the back and sides of the tongue (Hoon et al., 1999).

\section{Structure and Activation of the T1R}

Each subunit of the T1R is made up of three domains consistent with other class C Gcoupled proteins. The class C G-coupled proteins contain an extracellular amino-terminal domain which is connected to a seven transmembrane helical domain at the $\mathrm{C}$ terminus via a cysteine-rich domain (Pin et al., 2003). The amino-terminal domain of the sweet receptor is also often called the extracellular venus-flytrap (VFT) domain (Fernstrom et al., 2012).

The T1R2 and T1R3 subunits are most commonly activated by ligand binding at the VFT domain (Li et al., 2002; Kuhn et al., 2004; Xu et al., 2004; Jiang et al., 2005b; Nie et al., 2005; Chandrashekar et al., 2006; Masuda et al., 2012; Shrivastav \& Srivastava, 2013; Mayank, 2015). The VFT domain is folded with an open cleft, or open conformation, when not interacting with a ligand. When a sweet stimuli enters the folded area and interacts with the corresponding amino acids, the cleft closes (Kunishima et al., 2000; Pin et al., 2003; Shrivastav \& Srivastava, 2013). The closed conformation is more stable than the open conformation (Kunishima et al., 2000; Pin et al., 2003). Not only does the interaction stabilize the receptor, it has also been speculated that the orientation of the T1R changes so the T1R2 and T1R3 VFT domains approach and eventually touch each other. This may destabilize the conformation of the two closed VFT domains (Pin et al., 2003).

The activation of the T1R is initiated when sweet stimuli interact with specific amino acid residues in the T1R2 and/or T1R3 subunit proteins. Twenty-two amino acid residues are in close proximity to the most common binding site, the cleft of the VFT domain. The residues are 
Y103, D142, N143, S144, S165, A166, I167, S168, Y215, R270, V272, V274, F275, S301, E302, S303, A305, T326, R378, L379, S380, and R383 (Li et al, 2011). While some of these residues directly interact with sweet molecules, others, including Y103, R378, and R383, have been proposed to be residues that interact with other residues in the closed conformation. Other residues that may participate in interactions with residues are A43, K65, I67, P277, D278, L279, D307, and V309 (Zhang et al., 2010). These residues have been named 'pincer residues' and act to stabilize the closed conformation of the VFT domain (Table 1).

The activation of taste receptors triggers the transmission of basic taste information from the oral cavity, through sensory nerve fibers, to the brain. Signals from the fungiform papillae are transmitted through the chorda tympani, while the circumvallate and foliate papillae use the glossopharyngeal nerve (Frank et al., 1991 Zhang et al., 2003; Yarmolinsky et al., 2009). When the basic taste information, along with aromatic, physical, and chemesthetic information, is processed by the brain, the sensory characteristics of the food is experienced.

\section{Binding Sites of the T1R}

Sweet tastants display a wide variety of structures. Traditional mono- and disaccharides, such as sucrose, glucose, and fructose, have relatively simple structures compared to the various high potency sweeteners, which can be much larger and contain different functional groups. The T1R is activated by a variety of sweet tastants because the T1R 2 and T1R 3 subunits each contain multiple sites with which sweet molecules can interact (Kuhn et al., 2004; Xu et al., 2004; Nie et al., 2005; Chandrashekar et al., 2006; Yarmolinsky et al., 2009; Treesukosol et al., 2011; Masuda et al., 2012; Shrivastav \& Srivastava, 2013; Mayank, 2015; Appendix A).

Cell culture techniques were utilized in early T1R research to determine the binding domain and important amino acid residues for each sweet tastant (Li et al., 2002; Xu et al., 2004; 
Jiang et al., 2005b; Nie et al., 2005). Cell culture research involves extracting cells from animal tissues, primarily from mice, subcloning into a vector cell, and proliferating the cells on a growth medium. Mutagenesis of a specific residue with PCR techniques allows for the evaluation of one residue at a time. Once the cell line becomes stable, assays can be performed that measure the activation of the receptor. Most commonly, the identification of released calcium identifies cellular activation via a change in fluorescence of a calcium indicator dye (Masuda et al., 2012). The results of cell culture research have shown the VFT domain of the T1R2 and T1R3 subunits is the binding site for a large group of sweet tastants. This group includes sucrose, fructose, and glucose (Nelson et al., 2001; Li et al., 2002; Nie et al., 2005; Chandrashekar et al., 2006; Zhang et al., 2010), as well as artificial and natural high potency sweeteners, such as sucralose, acesulfame K (AceK), saccharin, stevioside, and rebaudiosides (Li et al., 2002; Kuhn et al., 2004; Nie et al., 2005; Zhang et al., 2010; Masuda et al., 2012; Mayank, 2015). Other high potency sweeteners only interact with the VFT domain of the T1R2 domain. This is the case for aspartame and neotame (Xu et al., 2004; Jiang et al., 2005b; Nie et al., 2005; Shrivastav \& Srivastava, 2013). Other domains of the T1R2 and T1R3 are also available for interaction with high potency sweeteners. For example, the seven transmembrane domain of the T1R3 receptor is the binding site for cyclamate, an artificial sweetener (Fujiwara et al., 2012; Shrivastav \& Srivastava, 2013; Xu et al., 2004). Lactisole, a sweetness inhibitor, has also been shown to interact with the seven transmembrane domain of the T1R3 (Jiang et al., 2005a; Shrivastav \& Srivastava, 2013). Some proteins may also be perceived as sweet and interact with the VFT domain, as is the case with neoculin and brazzein, or the cysteine-rich domain, as with thaumatin and monellin (Jiang et al., 2004; Assadi-Porter et al., 2010; Koizumi et al., 2007; Masuda et al., 2013; Ohta et al., 2011). 
The limitation with early cell culture research is that the main purpose was to identify the subunit and domain with which sweet molecules interact. In reality, determining each amino acid that a sweet molecule interacts with gives the most information about the interaction mechanism. Evaluating a large number of amino acid residues with cell culture techniques is difficult, because the researcher needs to mutate one amino acid residue at a time in order to determine its importance in sweetness perception. Therefore, a large number of mutated cell lines would be needed to evaluate a large number of residues. However, a combination of cell culture research and molecular modeling may more completely and efficiently describe the binding interactions between sweet stimuli and the T1R.

Zhang et al. (2010) combined cell culture and molecular modeling techniques to characterize the modes of binding for sucrose and sucralose. Both sucrose and sucralose interact with D142, S144, I167, and E302 in the binding cleft region of the VFT domain. Sucralose forms additional interactions with proposed pincer residues, Y103 and P277, increasing the intensity of the response (Zhang et al., 2010).

Stevioside is a large molecule compared to sucrose and sucralose. The size of the molecule allows stevioside to interact with more residues than sucrose or sucralose (Zhang et al., 2010). However, researchers have proposed differing important residues for stevioside. Zhang et al. (2010) used cell culture and molecular modeling to show the primary residues for stevioside are at the cleft of the VFT domain, similar to sucrose and sucralose. Mayank (2015) used only computer molecular modeling techniques to identify nine total residues with which stevioside many interact in the T1R2 and T1R3, many of which occur outside the binding cleft of the VFT. Given the complexity of the molecule, the interaction between stevioside and the T1R may be a combination of both results. 
Unlike sucrose and sucralose, aspartame interacts with two different sites. The first site is at the binding cleft of the VFT, while the second is located away from the cleft, but still in the VFT (Li et al., 2011; Mallet et al., 2015). Three of the residues aspartame interacts with, D142, S144, and E302, are the same as sucrose and sucralose (Mallet et al., 2015; Masuda et al., 2012). However, four additional residues, that are either in or proximate to the binding cleft, add to the complexity of the aspartame interaction (Mallet et al., 2015). There are residues that have been shown to be critical for aspartame that have been hypothesized to be pincer residues (Li et al., 2011). It has also been proposed that two water molecules are also required to activate the receptor with aspartame (Mallet et al., 2015). The secondary binding site in the VFT domain is required for sweetness perception, but has a lower affinity for aspartame than the binding cleft site (Jiang et al., 2005b; Li et al., 2011). This large, complex binding mechanism creates a sweetness detection threshold that is roughly 78 times less than that for sucrose (Ottinger et al., 2003).

\section{Synergistic Enhancement of Sweetness}

High potency sweeteners interact with a large number of residues, resulting in a more intense sweetness perception. However, synergistic sweetness enhancement may also be possible when combining two sweet tastants, creating a more intense sweetness than the sum of the isolated molecules.

When sucrose is combined with a sweetness enhancer, SE-3, both molecules simultaneously interact with the binding cleft in the VFT domain (Zhang et al., 2010). The presence of the sweetness enhancer allows for interaction with four additional residues than those sucrose normally interacts with. This increases the stability of the closed conformation of the VFT domain and the perceived sweetness intensity. There is also a hydrogen bond formed 
between sucrose and the sweetness enhancer. The enhancement of sucralose with a sweetness enhancer, SE-2, has a similar mechanism as described with sucrose and SE-3 (Zhang et al., 2010). Sucralose and SE-2 simultaneously bind to the binding cleft in the VFT domain and interact with more residues than sucralose can alone. Neither SE-2 nor SE-3 exhibit sweetness when used on their own (Servant et al., 2010).

Research has also shown enhancement of sweetness from sucrose with neohesperidin dihydrochalacone (NHDC) or cyclamate. NHDC and cyclamate enhance sweetness by binding to the transmembrane domain of T1R3 while the primary sweetener binds at its normal site (Xu et al., 2004; Winnig et al., 2007; Fujiwara et al., 2012; Shrivastav \& Srivastava, 2013). Besides binding location, NHDC and cyclamate are further differentiated from SE-2 and SE-3 because NHDC and cyclamate both enhance the sweetness from tastants other than sucrose and sucralose, including neotame, rebaudiosdies, stevioside, aspartame, saccharin, and AceK (Fujiwara et al., 2012). In addition, NHDC and cyclamate are perceived as sweet when used alone.

Sweetness enhancement is a heavily researched area and this review is not meant to discuss the breadth of research in this area. However, the two examples discussed show multiple mechanisms through which the perception of sweetness can be synergistically enhanced using multiple sweeteners or sweetness enhancers. For a more detailed review of sweetness enhancement research, read Grenby, (1996), O'Donnell and Kearsley (2012), DuBois and Prakash (2012), Varzakas et al. (2012), and O’Brien-Nabors (2012). 


\section{Chapter 3 - The Effect of Genetic Variation on Sensitivity to Sweet}

\section{Tastants}

\section{Genetic Variation of the T1R}

Each of the three subunits of the T1R are a protein encoded by specific genes. In humans, the genes that encode T1R1, T1R2, and T1R3 are called TAS1R1, TAS1R2, and TAS1R3, respectively. The nomenclature differs for mice and rat genes as they are Tas $1 r 1, \operatorname{Tas} 1 r 2$, and Tas1r3 (Bachmanov \& Beauchamp, 2007). The genes that encode sweet taste receptors are located in chromosome 1 in humans and chromosome 4 in mice. Each of the subunit proteins of the human and mouse T1R are encoded by at least five exons (Liao \& Schultz, 2003). Alternative splicing is also believed to be important in the final protein, potentially affecting which exons are used in the final mRNA (Bachmanov \& Beauchamp, 2007).

Genetic variation in TAS1R genes may affect the amino acid sequence of the T1R subunit proteins, potentially effecting the function of the receptor (Kim et al., 2006). Hundreds of potential single nucleotide polymorphisms in the TAS1R genes have been found (Kim et al., 2006; Fushan et al., 2009 Fushan et al., 2010; Haznedaroğlu et al., 2015; Dias et al., 2015). Single nucleotide polymorphisms can occur in many or a limited number of geographic populations (Kim et al., 2006; Fushan et al., 2010). Only a fraction of these variates result in amino acid changes in the T1R. Some of these variates may affect amino acids near the binding cleft of the VFT domain, but the amino acids in the binding cleft appear to be very conserved (Kim et al., 2006).

The TAS1R2 gene has an unusual amount of variation compared to other genes (Kim et al., 2006). The majority of the T1R2 variation occurs in the VFT domain of the T1R (Kim et al., 2006), the site where many sweet molecules bind. It is interesting to note that the majority of the 
variation in the bitter receptors occur in the transmembrane domain, which is the main interaction site for bitter compounds (Kim et al., 2006).

\section{Effect of Genetic Variation on Sensitivity to Tastants Received by G-Coupled}

\section{Protein Receptors}

Research has shown genetic variation in receptor genes can affect sensitivity to umami and bitter tastants, which, like sweet tastants, are also detected by G-coupled protein receptors. For example, a few single nucleotide polymorphisms in the TAS1R3 have been linked to differences in umami sensitivity to L-glutamate (Chen et al., 2009; Shigemura et al., 2009). Furthermore, single nucleotide polymorphisms (SNP) in the TAS2R can also affect the perception of bitterness from products as diverse as AceK and vegetables (Allen et al., 2013; Sandell \& Breslin, 2006). There has also been a variety of research with differing results on the relationships between sensitivity to bitter compounds, such as 6- $n$-propylthiouracil (PROP) and phenylthiocarbamide (PTC), and sensitivity to other bitter compounds (Allen et al., 2013;

Sandell \& Breslin, 2006; Tepper et al., 2009). Some studies have found heightened sensitivity to PROP or PTC may be associated with heightened sensitivity to other bitter compounds, while other studies have not. Although these studies were not conducted with sweet tastants, it does generally indicate that genetic variation in genes for G-coupled protein receptors may affect sensitivities to a taste quality. In addition, the variation in sensitivity may affect specific classes of compounds within a taste quality, while others remain more consistent.

In addition to the research on bitter an umami sensitivity, there is one study that connects sweet tastant sensitivity and genetic variation in the TAS1R genes. In this study, one SNP in the TAS1R2 was associated with both sweet taste and sugar intake (Dias et al., 2015). Those with a $\mathrm{BMI} \geq 25$ and a $\mathrm{G}$ allele for the rs12033832 SNP displayed decreased sensitivity to sucrose. 
Conversely, those with a BMI $<25$ and a G allele for the rs12033832 SNP displayed increased sensitivity to sucrose. The difference in the sensitivity effect between the two groups may be explained by differing levels of leptin resistance between obese and normal weight individuals and the effect of leptin on signal transduction (Dias et al., 2015). Previous research has shown the rs12033832 SNP is a prevalent variation in many populations (Kim et al., 2006; Fushan et $a l ., 2009)$. There is also evidence that suggests other genetic variation can affect overall sweetness perception. Two SNPs in non-coding regions of TAS1R3, identified by rs307355 and rs35744813, were shown to affect gene transcription and result in lower sweetness sensitivity. Typically, these two positions are C/C. A T/C genotype reduces sweetness perception by $25 \%$ and a $\mathrm{T} / \mathrm{T}$ genotype by $50 \%$. The proportion of people exhibiting $\mathrm{C} / \mathrm{T}$ or $\mathrm{T} / \mathrm{T}$ genotypes varies greatly by region with $60 \%$ of people exhibiting one of these genotypes in an African population, but $9 \%$ in a European population (Fushan et al., 2009). In another study, differences in sucrose sensitivity arose from variation in genes that encode gustducin, a signal protein within the taste receptor (Fushan et al., 2010).

Although only one study has found an association between TAS1R genetic variation and sweetness sensitivity, other studies have shown an association between TAS1R variation and human behavior. In overweight or obese subjects, those that carried the recessive TAS1R2 gene for V191 instead of I191 consumed less sugar per day before and after nutritional education (Eny et al., 2010). In addition, some genetic variations in TAS1R2 and TAS1R3 were also associated with increased risk of dental caries in children aged seven to twelve (Haznedaroğlu et al., 2015). 


\section{Genetic Variation and its Effect on Person-to-Person Sweetness Intensity Variability of Sweeteners}

The research indicating a relationship with TAS1R variation and sweetness sensitivity has not done so with different categories of sweet tastants. The effect of individual genetic variation on sensitivity to specific sugars, artificial, and natural sweeteners has not been observed. However, since some amino acid residues in the T1R may be more susceptible to genetic variation than others, binding sites of certain sweet tastants may be more susceptible to genetic variation than others. Because changes in amino acid residues in T1R2 and T1R3 may affect sweetness sensitivity, some classes of sweeteners may have higher person-to-person sweetness sensitivity variation due to more genetic variation in the corresponding binding site.

Differences in subject-to-subject sensitivity variation between sweeteners have been observed in studies using mice. For example, a mutation in the I60 residue is one of the key genetic variations that is thought to result in the lower sweetness sensitivity experienced by mice compared to humans (Nie et al., 2005). The I60 residue is located in the secondary binding site that has been proposed for aspartame (Jiang et al., 2005b; Li et al., 2011). Genetic variations, most likely at the I60 residue, have also been shown to cause behavioral differences in mice to many sweet stimuli solutions, but not all sweet solutions (Inoue et al., 2007). Tas 1r variation may also cause a phenotypic division among mice in which there is a group of sucralose avoiders $(\sim 75 \%)$ and sucralose likers ( 25\%), while neither avoid sucrose (Loney et al., 2014). This means the genetic variation in the T1R may be capable of effecting a fraction of the sweet tastants.

However, research with human subjects measuring sweetness intensity does not show different levels of person-to-person sweetness intensity variation for specific classes of sweet 
tastants. This is the case when investigating model soft drinks, milk, coffee, and an Indian snack food (Choi \& Chung, 2015; Patil et al., 2014; Thai et al., 2011; Zhao \& Tepper, 2007).

Furthermore, some researchers do not publish the variation on sweetness intensities, making it impossible to determine a difference in person-to-person sweetness intensity variation (Li et al., 2015; Morais et al., 2014; Waldrop \& Ross, 2014).

Although many human analyses do not show person-to-person variation differences between sweeteners, human methods may not be sensitive enough to determine such differences. Furthermore, human testing may be a biased method for the investigation of variation in sweetness delivery. The off-flavors in many high potency sweeteners may cause confusion and bias in an experiment focused on sweet intensity variability into showing increased variability for high-potency sweeteners (Allen et al., 2013; Kuhn et al., 2004; Li et al., 2015).

Cell culture may provide a method that is more sensitive and less biased for the measurement of sweetness intensity variation than human measurements. Li et al. (2002) measured cell culture responses to different sweeteners. The percentage of responding cells to aspartame, cyclamate, saccharin, and AceK showed higher variability than the response to sucrose, fructose, and glucose. Furthermore, even though sucralose is an artificial sweetener, it had a low variation in cellular response that was similar to sucrose. This may be because the binding mechanism of sucralose is similar to sucrose (Zhang et al., 2010). Fujiwara et al. (2012) also observed an increased cellular response variation for binary mixtures of sucrose with AceK and cyclamate compared to control sucrose solutions. Furthermore, the response of cells to sucralose was less variable than to AceK, stevioside, and rebaudiosides (Fujiwara et al., 2012). Data from Masuda et al. (2012) showed sucralose, with similar binding mechanisms as sucrose, had a lower variation in cellular response than aspartame, AceK, and cyclamate in a wild-type 
cell line. A limited number of studies did not show a noticeable difference in cellular response variation between sucrose and other high potency sweeteners (Servant et al., 2010) These findings suggest there may be increased variability in the perception of high potency sweeteners based on inconsistent receptor activation or genetic variation within the wild-type cell lines. 


\section{Chapter 4 - Discussion and Conclusion}

A wide range of molecular structures are perceived as sweet due to the many unique binding interaction patterns between sweet tastants the T1R receptor. Binding interactions occur between sweet molecules and specific amino acid residues within the domains of the T1R2 and T1R3 subunits. Since each residue within the T1R has a different predisposition to genetic variation based on the TAS1R, the binding sites for sweet tastants may also have different predispositions to variation. Some residues, such as those near the VFT binding cleft of the T1R2 and T1R3, appear to be more conserved in humans, while those in other regions of the receptor may be more susceptible to genetic variation (Li et al., 2011).

A growing body of research indicates a relationship between genetic variation and sweetness sensitivity. Genetic variation in the TAS1R, as well as other genetic variation affecting transcription and signaling proteins, may affect sensitivity to sweet tastants (Fushan $e t$ al., 2009; Fushan et al., 2010; Dias et al., 2015). Furthermore, there may be a relationship between genetic variation and human and mouse behavior around sweet tastants (Eny et al., 2010; Haznedaroğlu et al., 2015; Dias et al., 2015). Other evidence suggests genetic variations in the genes for G-coupled protein receptors may affect specific classes of tastants within a taste quality (Sandell \& Breslin, 2006; Inoue et al., 2007; Tepper et al., 2009; Allen et al., 2013; Loney et al., 2014). Furthermore, cell culture studies indicate increased variability in cellular response for high-potency sweeteners with binding sites away from the binding cleft of the VFT, such as aspartame, stevioside, cyclamate, saccharin, and AceK (Fujiwara et al., 2012; Li et al., 2002; Masuda et al., 2012). This may be due to inconsistent activation of the receptor or variation within the wild-type cell lines. 
Despite a variety of research indicating that genetic variation may affect the perceived sweetness intensity from some sweet tastants more than others based on binding site interaction and TAS1R variation, experiments with human subjects do not show categories of sweet tastants with higher variation of person-to-person sweetness intensity than others (Zhao \& Tepper, 2007; Choi \& Chung, 2015; Patil et al., 2014; Thai et al., 2011). Experiments measuring sweet intensities to specific sweet tastants may not have been conducted with populations with wide enough diversity, because many of the genetic differences in the TAS1R are linked to specific geographical regions and might not be detected if the sampled participants have ancestors from a similar location (Kim et al., 2006; Fushan et al., 2010). Furthermore, human testing methods may be not be sensitive enough to detect differences in sweetness intensity variation or could potentially be biased.

It can be proposed that utilizing sweeteners that bind with, and only with, the cleft of the VFT domain in the T1R2 and T1R3 subunits of the T1R should result in a more consistent delivery of sweetness intensity across a population of genetically diverse individuals. Because humans have evolved to detect sugars as a source of energy with VFT binding cleft residues, these residues are under more evolutionary pressure to remain conserved (Chandrashekar et al., 2006; Li et al., 2011). Other residues in the T1R2 and T1R3 were not as important in the detection of sugars, making them more susceptible to genetic variation. The sweeteners that interact at the VFT domain of the T1R2 and T1R3 include traditional bulk sweeteners, such as sucrose, glucose, and fructose, and the high-potency sweetener sucralose. Sweeteners that interact with other sites may deliver a more variable sweetness intensity due to genetic variation present in the population or inconsistent receptor activation. Based on binding interaction site information and cell culture response variation data, AceK, aspartame, and saccharin may have 
moderately more person-to-person variation compared to sugars and sucralose, while cyclamate, stevioside, and rebaudiosides may have considerably more person-to-person variation.

The implication of this hypothesis is that when developing food products intended for a population of genetically diverse people, optimization for the entire population may be difficult with high-potency sweeteners that behave inconsistently between people with varied genetic backgrounds. Therefore, utilizing sweeteners with a more consistent delivery of sweetness intensity should improve food product optimization for products intended for an entire population of genetically diverse people.

Future research in this area should expand upon the knowledge of binding interactions with the T1R using a combination of cell culture and computer modeling techniques. Furthermore, a comparison of sensitivities to specific sweet tastants at threshold and/or suprathreshold levels, such as in Diaz et al. (2015), with genetic variation in the TAS1R should be made to determine if variations effect sensitivity to specific sweet tastants individually. 


\section{References}

Adler, E., Hoon, M., Mueller, K., Chandrashekar, J., Ryba, N. \& Zuker, C. (2000). A novel family of mammalian taste receptors. Cell, 100(6), 693-702.

Allen, A., McGeary, J., Knopik, V. \& Hayes, J. (2013). Bitterness of the non-nutritive sweetener acesulfame potassium varies with polymorphisms in TAS2R9 and TAS2R31. Chemical Senses, 38(5), 379-389.

Assadi-Porter, F. M., Maillet, E. L., Radek, J. T., Quijada, J., Markley, J. L., \& Max, M. (2010). Key amino acid residues involved in multi-point binding interactions between Brazzein, a sweet protein, and the T1R2-T1R3 human sweet receptor. Journal of Molecular Biology, 398(4), 584-599.

Bachmanov, A. \& Beauchamp, G. (2007). Taste receptor genes. Annual Review of Nutrition, 27, 389-414.

Chandrashekar, J., Hoon, M., Ryban N. \& Zuker, C. (2006). The receptors and cells for mammalian taste. Nature 444(16), 288-294.

Chandrashekar, J., Kuhn, C., Oka, Y., Yarmolinsky, D., Hummler, E., Ryba, N. \& Zuker, C. (2010). The cells and peripheral representation of sodium taste in mice. Nature, 464, 297 302.

Chandrashekar, J., Mueller, K, Hoon, M, Adler, E., Feng, L, Guo, W., Zuker, C. \& Ryba, N. (2000). T2Rs function as bitter taste receptors. Cell, 100(6), 703-711.

Chen, Q., Alarcon, S., Tharp, A., Ahmed, O., Estrella, N., Greene, T., Rucker, J. \& Breslin, P. (2009). Perceptual variation in umami taste and polymorphisms in TAS1R taste receptor genes. The American Journal of Clinical Nutrition, 90(3), 770S-779S.

Choi, J. \& Chung, S. (2015). Sweetness potency and sweetness synergism of sweeteners in milk and coffee systems. Food Research International, 74, 168-176.

Dasgupta, K., Singh, A. \& Ireland, W. (1990). Taste bud density in circumvallate and fungiform papillae of the bovine tongue. Histology and Histopathology, 5(2), 169-172.

Dias, A., Eny, K., Cockburn, M., Chiu, W., Nielsen, D., Duizer, L. \& El-Sohemy, A. (2015). Variation in the TAS1R2 gene, sweet taste perception and intake of sugars. Journal of Nutrigenetics and Nutrigenomics, 8(2), 81-90.

DuBois, G. \& Prakash, I. (2012). Non-caloric sweeteners, sweetness modulators, and sweetener enhancers. Annual Review of Food Science and Technology, 3, 353-380.

Eny, K., Wolever, T., Corey, P. \& El-Sohemy, A. (2010). Genetic variation in TAS1R2 (Ile191Val) is associated with consumption of sugars in overweight and obese individuals in 2 distinct populations. The American Journal of Clinical Nutrition, 92(6), 1501-1510. 
Fernstrom, J. D., Munger, S. D., Sclafani, A., De Araujo, I. E., Roberts, A. \& Molinary, S. (2012). Mechanisms for sweetness. The Journal of Nutrition, 142(6), 1134S-1141S.

Frank, M. (1991). Taste-responsive neurons of the glossopharyngeal nerve of the rat. Journal of Neurophysiology, 65(6), 1452-63.

Fujiwara, S., Imada, T., Nakagita, T., Okada, S., Nammoku, T., Abe, K., \& Misaka, T. (2012). Sweeteners interacting with the transmembrane domain of the human sweet-taste receptor induce sweet-taste synergisms in binary mixtures. Food Chemistry, 130(3), 561-568.

Fushan, A. A., Simons, C. T., Slack, J. P., Manichaikul, A. \& Drayna, D. (2009). Allelic polymorphism within the TAS1R3 promoter is associated with human taste sensitivity to sucrose. Current Biology, 19(15), 1288-1293.

Fushan, A., Simons, C., Slack, J. \& Drayna, D. (2010). Association between common variation in genes encoding sweet taste signaling components and human sucrose perception. Chemical Senses, 35(7), 579-592.

Grenby, T. (1996). Advances in sweeteners (1st ed.). London ; New York, N.Y.: Blackie Academic \& Professional.

Haznedaroğlu, E., Koldemir-Gündüz, M., Bakır-Coşkun, H., Bozkus, H., Süsleyici-Duman, G. \& Mentes, A. (2015). Association of Sweet Taste Receptor Gene Polymorphisms with Dental Caries Experience in School Children. Caries Research, 49(3), 275-81.

Hoon, M. A., Adler, E., Lindemeier, J., Battey, J. F., Ryba, N., \& Zuker, C. S. (1999). Putative mammalian taste receptors: a class of taste-specific GPCRs with distinct topographic selectivity. Cell, 96(4), 541-551.

Huang, A., Chen, X., Hoon, M., Chandrashekar, J., Guo, W., Tränkner, D., Ryba, J. \& Zuker, C. (2006). The cells and logic for mammalian sour taste detection. Nature, 442, 934-938.

Inoue, M., Glendinning, J., Theodorides, M., Harkness, S., Li, X., Bosak, N., Beauchamp, G., \& Bachmanov, A. (2007). Allelic variation of the Tas1r3 taste receptor gene selectively affects taste responses to sweeteners: Evidence from 129.B6-Tas1r3 congenic mice. Physiological Genomics, 32(1), 82-94.

Ishimaru, Y., Inada, H., Kubota, M., Zhuang, H., Tominaga, M., \& Matsunami, H. (2006). Transient receptor potential family members PKD1L3 and PKD2L1 form a candidate sour taste receptor. Proceedings of the National Academy of Sciences of the United States of America, 103(33), 12569-12574.

James, WPT. (2009). WHO recognition of the global obesity epidemic. International Journal of Obesity, 32(S7), S120-S126.

Jiang, P., Ji, Q., Liu, Z., Snyder, L., Benard, L., Margolskee, R. \& Max, M. (2004). The cysteinerich region of T1R3 determines responses to intensely sweet proteins. The Journal of Biological Chemistry, 279(43), 45068-45075. 
Jiang, P., Cui, M., Zhao, B., Liu, Z., Snyder, L., Benard, L., Osman, R., Margolskee, R. \& Max, M. (2005a). Lactisole interacts with the transmembrane domains of human T1R3 to inhibit sweet taste. The Journal of Biological Chemistry, 280(15), 15238-15246.

Jiang, P., Cui, M., Ji, Q., Snyder, L., Liu, Z., Benard, L., Margolskee, R.F., Osman, R. \& Max, M. (2005b). Molecular mechanisms of sweet receptor function. Chemical Senses, 30(1), I17-I18.

Kim, M., Kusakabe, Y., Miura, H., Shindo, Y., Ninomiya, Y. \& Hino, A. (2003). Regional expression patterns of taste receptors and gustducin in the mouse tongue. Biochemical and Biophysical Research Communications, 312(2), 500-506.

Kim, U., Wooding, S., Riaz, N., Jorde, L., \& Drayna, D. (2006). Variation in the human TAS1R taste receptor genes. Chemical Senses, 31(7), 599-611.

Kinnamon, J., Henzler, D. \& Royer, S. (1993). HVEM ultrastructural analysis of mouse fungiform taste buds, cell types, and associated synapses. Microscopy Research and Technique, 26(2), 142-156.

Koizumi, A., Nakajima, K., Asakura, T., Morita, Y., Ito, K., Shmizu-Ibuka, A., Misaka, T., Abe, K. (2007). Taste-modifying sweet protein, neoculin, is received at human T1R3 amino terminal domain. Biochemical and Biophysical Research Communications, 358(2), 585589.

Kuhn, C., Bufe, B., Winnig, M., Hofmann, T., Frank, O., Behrens, M., Lewtschenko, T., Slack, J. P., Ward, C. D. \& Meyerhof, W. (2004). Bitter taste receptors for saccharin and acesulfame K. The Journal of Neuroscience, 24(45), 10260-10265.

Kunishima, N., Shimada, Y., Tsuji, Y., Sato, T., Yamamoto, M., Kumasaka, T., Nakanishi, S., Jingami, H. \& Morikawa, K. (2000). Structural basis of glutamate recognition by a dimeric metabotropic glutamate receptor. Nature, 407(6807), 971-977.

Li, X., Staszewski, L., Xu, H., Durick, K., Zoller, M. \& Adler, E. (2002). Human receptors for sweet and umami taste. Proceedings of the National Academy of Sciences of the United States, 99(7), 4692-4696.

Li, X., Bachmanov, A., Maehashi, K., Li, W., Lim, R., Brand, J., Beauchamp, G., Reed, D. Tahi, C. \& Floriano, W. (2011). Sweet taste receptor gene variation and aspartame taste in primates and other species. Chemical Senses, 36(5), 453-475.

Li, X., Lopetcharat, K. \& Drake, M. (2015). Parents' and children's acceptance of skim chocolate milks sweetened by monk fruit and stevia leaf extracts. Journal of Food Science, 80(5), S1083-S1092.

Liao, J. \& Schultz, P. (2003). Three sweet receptor genes are clustered in human Chromosome 1. Mammalian Genome, 14(5), 291-301. 
Loney, G., Eckel, L., Bales, W., Carbonell, J., Houpt, T. \& Spector, A. (2014). Individual Differences in Sensory Processing in the Rattus as Assessed through the Bimodal Preference Profile for the Artificial Sweetener Sucralose: Do Rats Have a 'sweet' Tooth?, ProQuest Dissertations and Theses.

Lopez-Jimenez, N., Cavenagh, M., Sainz, E., Cruz-Ithier, M., Battey, J. \& Sullivan, S. (2006). Two members of the TRPP family of ion channels, Pkd113 and Pkd211, are co-expressed in a subset of taste receptor cells. Journal of Neurochemistry, 98(1), 68-77.

Maillet, E., Cui, M., Jiang, P., Mezei, M., Hecht, E., Quijada, J., Margolskee, R., Osman, R. \& Max, M. (2015). Characterization of the binding site of aspartame in the human sweet taste receptor. Chem. Senses, 40(8), 577-586.

Masuda, K., Koizumi, A., Nakajima, K., Tanaka, T., Abe, K., Misaka, T., Ishiguro, M. \& Meyerhof, W. (2012). Characterization of the modes of binding between human sweet taste receptor and low-molecular-weight sweet compounds. PLOS ONE, 7(4), E35380.

Masuda, T., Taguchi, W., Sano, A., Ohta, K., Kitabatake, N. \& Tani, F. (2013). Five amino acid residues in cysteine-rich domain of human T1R3 were involved in the response for sweet-tasting protein thaumatin. Biochimie, 95(7), 1502-1505.

Matsunami, H., Montmayeur, J. \& Buck, L. (2000). A family of candidate taste receptors in human and mouse. Nature, 404(6778), 601-604.

Mayank, V. J. (2015). Interaction model of steviol glycosides from Stevia rebaudiana (Bertoni) with sweet taste receptors: A computational approach. Phytochemistry, 116, 12-20.

Morais, E.C., Morais, A.R., Cruz, A.G. \& Bolini, H.M.A. (2014). Development of chocolate dairy dessert with addition of prebiotics and replacement of sucrose with different highintensity sweeteners. Journal of Dairy Science, 97(5), 2600-2609.

Nelson, G., Hoon, M., Chandrashekar, J., Zhang, Y., Ryba, N. \& Zuker, C. (2001). Mammalian sweet taste receptors. Cell, 106(3), 381-390.

Nelson, G., Chandrashekar, J., Hoon, M., Feng, L., Zhao, G., Ryba, N. \& Zuker, C. (2002). An amino-acid taste receptor. Nature, 416(6877), 199-202.

Nie, Y., Vigues, S., Hobbs, J., Conn, G. \& Munger, S. (2005). Distinct contributions of T1R2 and T1R3 taste receptor subunits to the detection of sweet stimuli. Current Biology, 15(21), 1948-1952.

O’Brien-Nabors, L. (2012). Alternative sweeteners (4th ed.). Boca Raton, FL: CRC Press.

O'Donnell, K. \& Kearsley, M. (2012). Sweeteners and sugar alternatives in food technology (2nd ed.). Chichester, West Sussex ; Ames, Iowa: Wiley-Blackwell.

Ohta, K., Masuda, T., Tani, F., \& Kitabatake, N. (2011). The cysteine-rich domain of human T1R3 is necessary for the interaction between human T1R2-T1R3 sweet receptors and a 
sweet-tasting protein, thaumatin. Biochemical and Biophysical Research Communications, 406(3), 435-438.

Ottinger, H., Soldo, T. \& Hofmann, T. (2003). Discovery and structure determination of a novel Maillard-derived sweetness enhancer by application of the comparative taste dilution analysis (cTDA). Journal of Agricultural and Food Chemistry, 51(4), 1035-1041.

Patil, S., Ravi, R., Saraswathi, G. \& Prakash, M. (2014). Development of low calorie snack food based on intense sweeteners. Journal of Food Science and Technology, 51(12), 40964101.

Pin, J., Galvez, T. \& Prézeau, I. (2003). Evolution, structure, and activation mechanism of family 3/C G-protein-coupled receptors. Pharmacology and Therapeutics, 98(3), 325-354.

Sandell, M.A. \& Breslin, P. (2006). Variability in a taste-receptor gene determines whether we taste toxins in food. Current Biology, 16(18), R792-R794.

Servant, G., Tachdjian, C., Tang, X., Werner, S., Zhang, F., Li, X., Kamdar, P., Petrovic, G., Ditschun, T., Java, A., Brust, P., Brune, N. Dubois, G., Zoller, M. \& Karanewsky, D. (2010). Positive allosteric modulators of the human sweet taste receptor enhance sweet taste', Proceedings of the National Academy of Sciences of the United States of America, 107(10), 4746-4751.

Shigemura, N., Shirosaki, S., Sanematsu, K., Yoshida, R., Ninomiya, Y. \& Matsunami, H. (2009). Genetic and molecular basis of individual differences in human umami taste perception. PLoS ONE, 4(8), e6717.

Shrivastav, A. \& Srivastava, S. (2013). Human sweet taste receptor: complete structure prediction and evaluation. International Journal of Chemical and Analytical Science, 4, 24-32.

Tepper, B., White, E., Koelliker, Y., Lanzara, C., D'Adamo, P. \& Gasparini, P. (2009). Genetic variation in taste sensitivity to 6-n-propylthiouracil and its relationship to taste perception and food selection. Annals of the New York Academy of Sciences, 11701(1), 126-139.

Thai, P., Tan, E., Tan, W., Tey, T., Kaur, H., \& Say, Y. (2011). Sweetness intensity perception and pleasantness ratings of sucrose, aspartame solutions and cola among multi-ethnic Malaysian subjects. Food Quality and Preference, 22(3), 281-289.

Treesukosol, Y., Smith, K. R. \& Spector, A. C. (2011). The functional role of the T1R family of receptors in sweet taste and feeding. Physiology \& Behavior, 105(1), 14-26.

Varzakas, T., Labropoulos, A., \& Anestis, S. (2012). Sweeteners : Nutritional aspects, applications, and production technology. Boca Raton, FL: CRC Press.

Xu, H., Staszewski, L., Tang, H., Adler, E., Zoller, M., Li, X. \& Snyder, S. (2004). Different functional roles of T1R subunits in the heteromeric taste receptors. Proceedings of the National Academy of Sciences of the United States of America, 101(39), 14258-14263. 
Waldrop, M. \& Ross, C. (2014). Sweetener blend optimization by using mixture design methodology and the electronic tongue. Journal of Food Science, 79(9), S1782-S1794.

World Health Organization. (2003). Diet, nutrition and the prevention of chronic diseases report of a joint WHO/FAO expert consultation. Geneva: World Health Organization.

Winnig, M., Bufe, B., Kratochwil, N., Slack, J., \& Meyerhof, W. (2007). The binding site for neohesperidin dihydrochalcone at the human sweet taste receptor. BMC Structural Biology, 7, 66.

Yarmolinsky, D. A., Zuker, C. S., \& Ryba, N. (2009). Common sense about taste: from mammals to insects. Cell, 139(2), 234-244.

Zhang, F., Klebansky, B., Fine, R., Liu, H., Xu, H., Servant, G., Zoller, M., Tachdjian, C. \& Li, X. (2010). Molecular mechanism of the sweet taste enhancers. Proceedings of the National Academy of Sciences of the United States of America, 107(10), 4752-4757.

Zhang, Y., Hoon M., Chandrashekar, J., Mueller K., Cook, B., Wu, D., Zuker, C. \& Ryba, N. (2003). Coding of sweet, bitter, and umami tastes: different receptor cells sharing similar signalling pathways. Cell, 112(3), 293-301

Zhao, G., Zhang, Y., Hoon, M., Chandrashekar, J., Erlenbach, I., Ryba, N. \& Zuker, C. (2003). The receptors for mammalian sweet and umami taste. Cell, 115(3), 255-266.

Zhao, L. \& Tepper, B. J. (2007). Perception and acceptance of selected high-intensity sweeteners and blends in model soft drinks by propylthiouracil (PROP) non-tasters and super-tasters. Food Quality and Preference, 18(3), 531-540. 


\section{Appendix A - T1R Binding Interaction Summary}

Summary of Binding Domains and Important Amino Acid Residues in the

\section{T1R}

\begin{tabular}{|c|c|c|c|}
\hline Sweetener & Binding Domain & $\begin{array}{l}\text { Important Amino Acid } \\
\text { Residues in both the } \\
\text { T1R2 and T1R3 }\end{array}$ & Reference \\
\hline Acesulfame K & T1R2 +T1R3, VFT & $\begin{array}{l}\text { D142*, S144, D278P, } \\
\text { E302*, E382, R383*P }\end{array}$ & $\begin{array}{l}\text { Masuda } \text { et al., 2012; } \\
\text { Kuhn } \text { et al., } 2004\end{array}$ \\
\hline Aspartame & $\begin{array}{l}\text { T1R2, VFT Cleft + } \\
\text { Secondary Site }\end{array}$ & $\begin{array}{l}\text { Primary binding site: } \\
\text { S40, Y103*P, D142*, } \\
\text { S144*, S165*, S168*, } \\
\text { Y215*, D278P, E302*, } \\
\text { D307, R383*P } \\
\text { Secondary binding site: } \\
\text { F27, L41, H42, K60, E61, } \\
\text { Y62, E63, Y69, E340, } \\
\text { W341, R352, S354, Q355, } \\
\text { S356 }\end{array}$ & $\begin{array}{l}\text { Xu et al., 2004; Nie et } \\
\text { al., 2005; Jiang et al., } \\
\text { 2005b; Masuda et al., } \\
\text { 2012; Mallet } \text { et al., } \\
2015\end{array}$ \\
\hline Cyclamate & T1R3, 7TM & $\begin{array}{l}\text { Q636, S640, H641, P643 } \\
\text { L644, T645, H721, R723, } \\
\text { F730, H734, A735, T736, } \\
\text { L740, Y771, F772, W775, } \\
\text { V776 F778, V779 L782, } \\
\text { R790, Q794, I805 }\end{array}$ & $\begin{array}{l}\text { Fujiwara et al., 2012; } \\
\text { Jiang } \text { et al., 2005a; } \\
\text { Shrivastav \& Srivastava, } \\
2013\end{array}$ \\
\hline Neotame & T1R2, VFT & $\begin{array}{l}\text { F53, V56, P57, S144*, } \\
\text { Y218, E302*, P308, } \\
\text { V309 , H311, N312, } \\
\text { S356, Y357, I376, L377, } \\
\text { R378*, N460 }\end{array}$ & $\begin{array}{l}\text { Xu et al, 2004; } \\
\text { Shrivastav \& Srivastava, } \\
2013\end{array}$ \\
\hline
\end{tabular}




\begin{tabular}{|c|c|c|c|}
\hline Rebaudiosides & T1R2/T1R3, VFT & $\begin{array}{l}\text { N42, N44, G47, L51, N52, } \\
\text { R56, S104, E105, E148, } \\
\text { D215*, D216, E217, } \\
\text { R247, K255, D258, I325, } \\
\text { T326*, S336, P343, A345, } \\
\text { G346 }\end{array}$ & Mayank, 2015 \\
\hline Saccharin & T1R2 +T1R3, VFT & $\begin{array}{l}\mathrm{D} 142 *, \mathrm{~S} 144 *, \mathrm{D} 278^{\mathrm{P}} \\
\mathrm{E} 302 *, \mathrm{E} 382, \mathrm{R} 383 * \mathrm{P}\end{array}$ & $\begin{array}{l}\text { Masuda et al., 2012; } \\
\text { Kuhn et al., } 2004\end{array}$ \\
\hline Stevioside & $\begin{array}{l}\text { T1R2 +T1R3, VFT } \\
\text { and/or other VFT } \\
\text { residues }\end{array}$ & $\begin{array}{l}\text { Binding Cleft and/or N44, } \\
\text { R52, R56, E105, D216, } \\
\text { E217, K255, I352, A354 }\end{array}$ & Mayank, 2015 \\
\hline Sucralose & T1R2 +T1R3, VFT Cleft & $\begin{array}{l}\mathrm{Y} 103^{* \mathrm{P}}, \mathrm{D} 142^{*}, \mathrm{~S} 144^{*}, \\
\mathrm{I} 167^{*}, \mathrm{P} 277^{\mathrm{P}}, \mathrm{D} 278^{\mathrm{P}} \\
\mathrm{E} 302^{*}, \mathrm{D} 307^{\mathrm{P}}\end{array}$ & $\begin{array}{l}\text { Nie et al., 2005; Masuda } \\
\text { et al., 2012; Zhang et al., } \\
2010\end{array}$ \\
\hline Sucrose & T1R2 +T1R3, VFT Cleft & $\begin{array}{l}\text { D142*, S144*, I167*, } \\
\text { E302* }\end{array}$ & Zhang et al., 2010 \\
\hline
\end{tabular}

* indicates a residue at the binding cleft

${ }^{\mathrm{P}}$ indicates a proposed pincer residue (Zhang et al., 2010) 